\section{Circulating cell-free BRAF V600E during chemotherapy is associated with prognosis of children with Langerhans cell histiocytosis}

Langerhans cell histiocytosis (LCH), a rare neoplasm predominantly affecting young children, is characterized by accumulation of abundant $\mathrm{CD} 1 \mathrm{a}^{+} \mathrm{CD} 207^{+}$histiocytes with inflammatory lesions. ${ }^{1}$ Recurrent $B R A F$ V600E mutations have been identified in approximately $50 \%$ of LCH patients, which are correlated with high-risk features of $\mathrm{LCH}$ and increased resistance to the first-line therapy. ${ }^{2,3}$ Several recent studies have indicated cell-free (cf) BRAF V600E analysis in plasma can serve as a promising biomarker in $\mathrm{LCH} .{ }^{4,5}$ However, the prognostic significance of $\mathrm{cfBR} A F \mathrm{~V} 600 \mathrm{E}$ during chemotherapy remains to be proven. In this study, we monitored $\mathrm{cf} B R A F \mathrm{~V} 600 \mathrm{E}$ at different time points during chemotherapy in children with $\mathrm{LCH}$, and evaluated the correlation of the dynamics of cfBRAF V600E with patients' clinical outcomes.

Of the 151 consecutive patients with newly diagnosed LCH (age $<18$ years) who were included in the Beijing Children's Hospital LCH registry from February 2017 to June 2018, 102 patients with available biopsy and plasma samples were enrolled in this study (Online Supplementary Figure S1). The patients were treated with a systemic chemotherapy regimen based on LCH-III and LCH-S2005 protocols (Online Supplementary Table S1)..$^{6-8}$ Genomic DNA (gDNA) was extracted from unstained sections of paraffin-embedded tissue at diagnosis. Serial blood samples were collected from the patients at five time points: the time of diagnosis, week 6 (after the first

Table 1. Clinical characteristics of 81 patients with BRAF V600E positive Langerhans cell histiocytosis (LCH) according to cell-free BRAF $\mathrm{V} 600 \mathrm{E}$ detection at diagnosis.

\begin{tabular}{|c|c|c|c|c|}
\hline \multirow[t]{2}{*}{ Variables } & \multirow[t]{2}{*}{ Total n } & \multicolumn{2}{|c|}{ Cell-free BRAF V600E at diagnosis } & \multirow[t]{2}{*}{$\mathbf{P}$} \\
\hline & & Negative n (\%) & Positive n (\%) & \\
\hline Total & 81 & $26(32.1)$ & $55(67.9)$ & \\
\hline \multicolumn{5}{|l|}{ Sex } \\
\hline Male & 42 & $12(28.6)$ & $30(71.4)$ & \multirow[t]{2}{*}{0.634} \\
\hline Female & 39 & $14(35.9)$ & $25(64.1)$ & \\
\hline \multicolumn{5}{|l|}{ Age(years) at diagnosis, } \\
\hline$<3$ years & 56 & $13(23.2)$ & $43(76.8)$ & \multirow[t]{2}{*}{0.019} \\
\hline$\geq 3$ years & 25 & $13(52.0)$ & $12(48.0)$ & \\
\hline Median (range) & $1.5(0.2-11.6)$ & $3.3(0.3-11.6)$ & $1.3(0.2-11.1)$ & 0.038 \\
\hline \multicolumn{5}{|l|}{ Clinical classification } \\
\hline SS LCH & 33 & $20(60.6)$ & $13(39.4)$ & \multirow{3}{*}{$<0.001$} \\
\hline MS RO- LCH & 23 & $4(17.4)$ & $19(82.6)$ & \\
\hline $\mathrm{MS} \mathrm{RO}^{+} \mathrm{LCH}$ & 25 & $2(8.0)$ & $23(92.0)$ & \\
\hline \multicolumn{5}{|l|}{ Involvement } \\
\hline Bone & 71 & $23(32.4)$ & $48(67.6)$ & 1.000 \\
\hline Unifocal bone & 15 & $6(40.0)$ & $9(60.0)$ & \multirow[t]{2}{*}{0.541} \\
\hline Multifocal bone & 56 & $17(30.4)$ & $39(69.6)$ & \\
\hline Skin & 35 & $5(14.3)$ & $30(85.7)$ & 0.004 \\
\hline Skin SS LCH & 6 & $3(50.0)$ & $3(50.0)$ & \multirow[t]{2}{*}{0.026} \\
\hline Skin MS LCH & 29 & $2(6.9)$ & $27(93.1)$ & \\
\hline Liver & 21 & $2(9.5)$ & $19(90.5)$ & 0.013 \\
\hline Spleen & 13 & $1(7.7)$ & $12(92.3)$ & 0.052 \\
\hline Hematologic & 8 & 0 & $8(100.0)$ & 0.050 \\
\hline Pituitary & 9 & $1(11.1)$ & $8(88.9)$ & 0.259 \\
\hline Central nervous system & 3 & $0(0)$ & $3(100.0)$ & 0.547 \\
\hline Lung & 17 & $3(17.6)$ & $14(82.4)$ & 0.242 \\
\hline Lymph nodes & 10 & $3(30.0)$ & $7(70.0)$ & 1.000 \\
\hline Ear & 27 & $2(7.4)$ & $25(92.6)$ & 0.001 \\
\hline Eye & 16 & $4(25.0)$ & $12(75.0)$ & 0.565 \\
\hline Oral & 18 & $4(22.2)$ & $14(77.8)$ & 0.397 \\
\hline Thyroid & 4 & $1(25.0)$ & $3(75.0)$ & 1.000 \\
\hline Thymus & 2 & $1(50.0)$ & $1(50.0)$ & 0.542 \\
\hline Mediastinum & 3 & $1(33.3)$ & $2(66.7)$ & 1.000 \\
\hline \multicolumn{5}{|l|}{ Response at week $6^{*}$} \\
\hline $\mathrm{NAD} / \mathrm{AD}$ better & $31(43.1)$ & $13(65.0)$ & $18(34.6)$ & \multirow{3}{*}{0.030} \\
\hline $\mathrm{AD}$ intermediate & $25(34.7)$ & $6(30.0)$ & $19(36.5)$ & \\
\hline $\mathrm{AD}$ worse & $16(22.2)$ & $1(5.0)$ & $15(28.8)$ & \\
\hline 2-year progression-free survival (\%) & $52.2 \pm 6.3$ & $92.3 \pm 5.2$ & $34.7 \pm 7.3$ & $<0.001$ \\
\hline 2-year overall survival (\%) & $97.0 \pm 2.1$ & 100 & $95.6 \pm 3.0$ & 0.343 \\
\hline
\end{tabular}

*For 71 evaluable patients. SS: single-system; MS: multiple system; RO: risk organ; NAD: non-active disease; AD: active disease. 
A

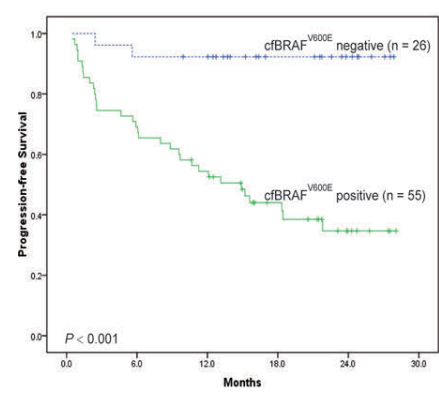

D

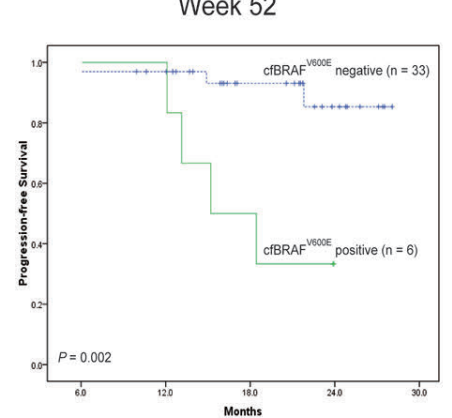

B

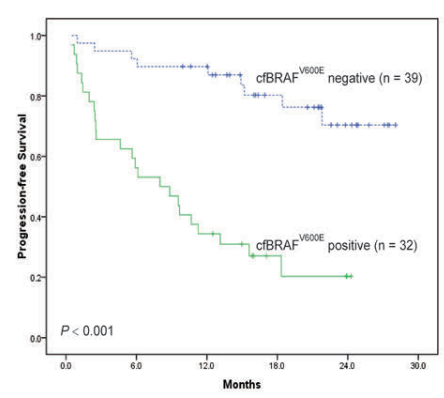

E

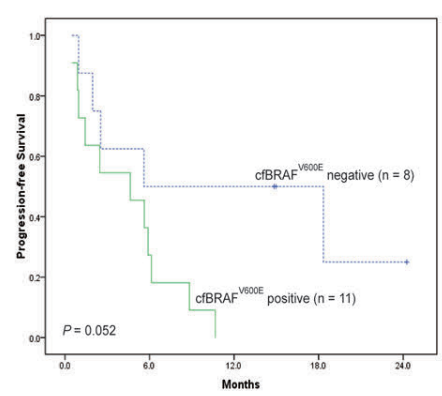

C

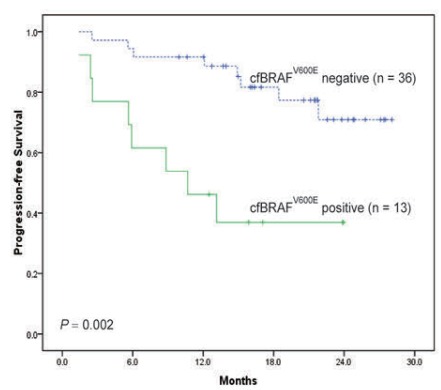

Figure 1. The prognostic significance of cfBRAF V600E at five time points during chemotherapy in children with Langerhans cell histiocytosis (LCH). (A) At diagnosis; (B) at week 6; (C) at week 12; (D) at week 52 of the first-line therapy; (E) at the end of course 8 of the second-line therapy.

initial induction therapy), week 12 (after the second initial induction therapy), week 52 (at the end of the maintenance treatment of the first-line therapy), and course 8 (at the end of the intensification treatment of the secondline therapy). Plasma cfDNA were isolated using the OIAamp Circulating Nucleic Acid Kit (Qiagen). BRAF V600E mutation in tissue gDNA or cfDNA was determined using OX200 ${ }^{\mathrm{TM}}$ Droplet Digital polymerase chain reaction system (Bio-Rad, Hercules, CA, USA) (Online Supplementary Table S2). The limit of the detection assay was determined at $0.1 \%$.

$B R A F$ V600E status in tissue samples was successfully determined in $117 \mathrm{LCH}$ patients. Among these, 88 $(75.2 \%)$ patients carried the BRAF V600E mutation in the tissue samples. There was no significant difference in survival of patients with or without the mutation (Online Supplementary Table S3). cf BRAF V600E in plasma was detected in 102 of the 117 patients at diagnosis, and was further monitored at four time points during chemotherapy in most of them (Online Supplementary Figure S1). There was no difference in the patients' characteristics, suggesting no substantial selection bias (Online Supplementary Table S4).

$\mathrm{cfBR} A F$ V600E at diagnosis was positive in $55(67.9 \%)$ of 81 patients with $B R A F$ V600E mutation in tissue samples. cfBRAF V600E was not identified in any of the 21 patients without the mutation in tissue samples. There was a relationship between $\mathrm{cfBR} A F \mathrm{~V} 600 \mathrm{E}$ and the disease features (Table 1). cfBRAF V600E was identified in $92.0 \%$ (23 of 25 ) of patients with multiple system (MS) $\mathrm{RO}^{+} \mathrm{LCH}, 82.6 \%$ (19 of 23 ) of patients with MS RO $\mathrm{LCH}$, and $39.4 \%$ (13 of 33 ) of patients with single system (SS) LCH $(P<0.001)$. The presence of cfBRAF V600E was correlated with age, liver involvement, hematologic system, ear or skin. Moreover, the 6 -week response rate was much lower in children with positive cfBRAF V600E than in those with negative detection $(34.6 \%$ vs. $65.0 \%$, $P=0.032$ ). Two-year progression-free survival (PFS) was much lower in the $\mathrm{cfBRAF} \mathrm{V} 600 \mathrm{E}$ positive group $(34.7 \% \pm 7.3 \%$ vs. $92.3 \% \pm 5.2 \%, P<0.001)$ (Figure $1 \mathrm{~A})$. Furthermore, the $13 \mathrm{SS}$ LCH patients with positive cfBRAF V600E obviously had worse PFS than the 20 SS patients with negative detection $(44.9 \% \pm 20.4 \%$ vs. $100 \%, P=0.003$ ) (Online Supplementary Figure S2A). Positive $\mathrm{cfBR} A F$ V600E had no significant impact on the $\mathrm{PFS}$ of the two groups in $\mathrm{MS} \mathrm{RO}^{-}$or $\mathrm{RO}^{+}$patients (Online Supplementary Figure $S 2 B$ and $C$ ). In a multivariate analysis, $\mathrm{cfBRAF}$ V600E at diagnosis remained an independent prognostic factor for PFS in childhood LCH (HR: 5.263, 95\%CI: 1.134-24.425, $P=0.034$ ) (Online Supplementary Table S5).

Thirty-two out of $71(45.1 \%)$ patients with available plasma samples at week 6 had detectable cfBRAF V600E. cfBRAF V600E at week 6 was also closely related to the treatment response (6-week response rate: $28.1 \%$ vs. $56.4 \%, P=0.005)$ and PFS $(20.3 \% \pm 8.4 \%$ vs. $70.4 \% \pm 9.0 \%$, $P<0.001$ ) (Figure 1B). cfBRAF V600E was quantified in 49 patients at week 12 . Thirteen patients $(26.5 \%)$ had positive detection. Similarly, The PFS of patients with positive $\mathrm{cfBRAF}$ V600E at week 12 was lower than that of negative group $(36.9 \% \pm 13.8 \%$ vs. $70.9 \% \pm 9.4 \%$, $P=0.002$ ) (Figure 1C).

$\mathrm{cfBR} A F$ V600E was sequentially monitored at four time points in 39 patients who were treated by the first-line therapy and determined at three time points in 19 patients treated with the second-line therapy (Figure 2A 


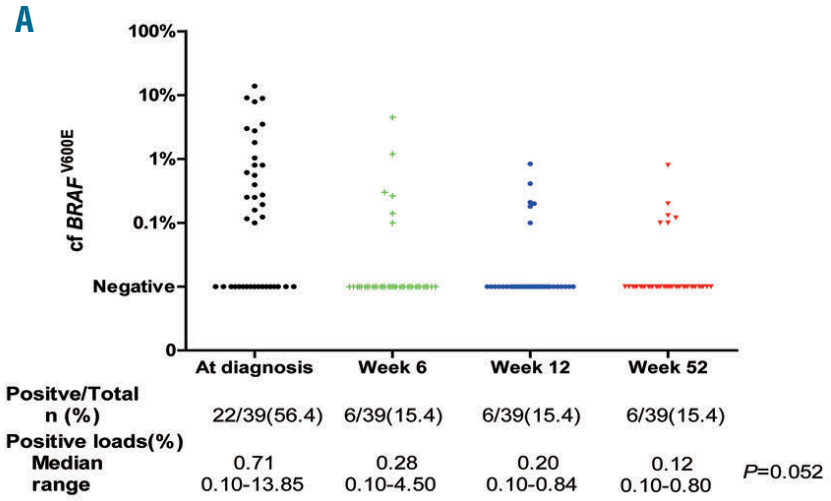

C

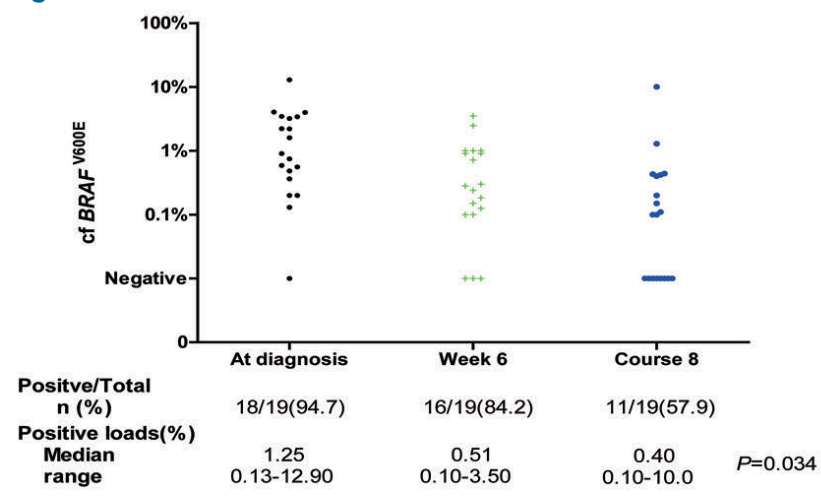

B

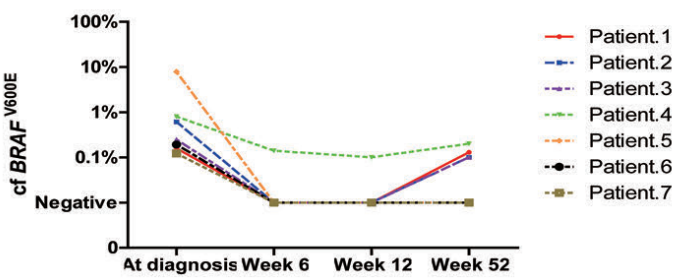

D

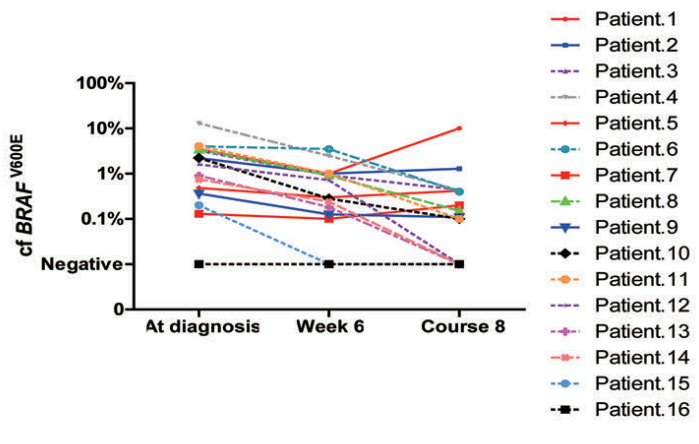

Figure 2. Sequential detection of cfBRAF V600E during follow up in children with Langerhans cell histiocytosis (LCH). (A) The levels of cfBRAF V600E at four time points in 39 patients treated by the first-line therapy. (B) Dynamics of cfBRAF V600E levels in seven relapsed patients treated by the first-line therapy. BRAF V600E reappeared at week 52 in Patients 1-3, while the mutation remained persistently detectable at all the four time points in Patient 4 . The cfDNA turned negative from week 6 to week 52 in Patients 5-7. (C) The levels of cfBRAF V600E at three time-points in 19 patients treated by the second-line therapy. (D) Dynamics of cfBRAF V600E in 16 relapsed patients treated by second-line therapy. Patients 1-11 had persistently positive detection from diagnosis to course 8. In particular, the levels of mutation obviously increased at course 8 in Patients 1, 5 and 7. Patients $12-15$ had negative detection at course 8 . Patient 16 had been negative for cfBRAF V600E since diagnosis.

and C). The PFS of the six patients with positive detection at week 52 was much worse than that of 33 patients with negative detection $(33.3 \% \pm 19.2 \%$ vs. $85.3 \% \pm 8.6 \%$, $P=0.002$ ) (Figure 1D). Notably, cfBRAF V600E detection turned from negative to positive at week 52 in three patients who relapsed 0-6 months later (Figure 2B, Patients 1-3; Online Supplementary Table S6). Eleven out of $19(57.9 \%)$ patients treated by the second-line therapy had persistently positive $\mathrm{cfBRAF}$ V600E detection from diagnosis to course 8 (Figure 2D, Patients 1-11) and relapsed, showing a tendency for worse PFS compared with other patients with negative detection (0 vs. $25.0 \% \pm 19.8 \%, \quad P=0.052$ ) (Figure $1 \mathrm{E}$ and Online Supplementary Table S6). In addition, cfBRAF V600E was positive at diagnosis in two out of $9(22.2 \%)$ SS patients who were not given any chemotherapy, and turned to negative detection 3-12 months later. The other seven children kept negative detection until one year. None of these patients had progression or relapse.

Detection of gene mutation in cfDNA is becoming a convenient and reliable tool for molecular testing, for new insights into tumor heterogeneity, and for monitoring residual disease. ${ }^{9}$ LCH has been recently redefined and treated as a myeloid neoplastic disorder, and progression or relapse has been the main cause of treatment failure. ${ }^{10}$ This study demonstrated that positive detection of $\mathrm{cfBR} A F \mathrm{~V} 600 \mathrm{E}$ at diagnosis and at the four subsequent time points during chemotherapy had a relevant impact on PFS of children with LCH.

In this study, cfBRAF V600E mutations at diagnosis were more frequently found in patients with MS LCH than in patients with SS disease. The presence of $\mathrm{cfBRAF}$ V600E was correlated with decreased PFS in SS LCH patients, but not in MS patients. The latter might be due to the fact that MS LCH patients were cfBRAF V600E positive in the majority of patients. A previous study showed that a French cohort had lower frequency of detectable cfBRAF V600E in patients with MS RO- LCH (41.7\% vs. $82.6 \%)$ and SS LCH (14.3\% vs. $39.4 \%)$ compared to this study (Online Supplementary Table S7). It should be noted that the patients in this study were younger at diagnosis and had more lung involvement or less hematologic involvement compared with those patients in the French study. The difference in the positive rate of $\mathrm{cfBR} A F \mathrm{~V} 600 \mathrm{E}$ might be due to the high heterogeneity of $\mathrm{LCH}$ and racial diversity.

Our findings showed that patients with $\mathrm{LCH}$ who remained or became $\operatorname{cf} B R A F$ V600E positive during chemotherapy were more likely to relapse. Thus, the dynamics of $\mathrm{cfBR} A F \mathrm{~V} 600 \mathrm{E}$ level during treatment should be closely followed. Moreover, it has been recently demonstrated that BRAF inhibitors have a dramatic effectiveness in treating patients with $B R A F$ V600E positive and refractory $\mathrm{LCH}$, and persistently positive $\mathrm{cf} B R A F$ 
V600E was associated with a higher reactivation risk after drug discontinuation. ${ }^{11}$ Future prospective studies with large sample size and long-term follow up were needed to confirm the prognostic significance of cfBRAF V600E detection.

It was reported that measurement of $B R A F$ V600E from whole blood was more accurate than cfDNA detection in several MS-LCH patients to measure disease activity. ${ }^{12}$ In addition, the levels of the BRAF V600E alleles in both cfDNA and whole blood were not totally consistent with the clinical condition of several $\mathrm{LCH}$ patients treated with the BRAF inhibitor. ${ }^{12,13}$ The most likely reason was that the BRAF V600E mutation was found in different cells, such as monocytes, dendritic cells, lymphocytes, or $\mathrm{CD} 34^{+}$hematopoietic cell progenitors. ${ }^{14}$ Precise monitoring of $B R A F$ V600E mutations in the specific cell subsets might be necessary in some patients in order to obtain accurate results.

In summary, this study showed that quantitative analysis of $c f B R A F$ V600E during chemotherapy had an impact on PFS of children with LCH. The challenge is to carefully integrate $\mathrm{cf} B R A F \mathrm{~V} 600 \mathrm{E}$ detection into clinical practice with the aim of improving outcome for these patients.

Lei Cui, ${ }^{1}$ Li Zhang, ${ }^{2}$ Hong-Hao Ma, ${ }^{2}$ Chan-Juan Wang, ${ }^{1}$ Dong Wang, ${ }^{2}$ Hong-Yun Lian, ${ }^{2}$ Wei-Jing Li, ${ }^{1}$ Qing Zhang, $\mathrm{Na} \mathrm{Li,}{ }^{1}$ Tian-You Wang, ${ }^{2}$ Zhi-Gang Li' and Rui Zhang ${ }^{2,3}$

'Laboratory of Hematologic Diseases, Beijing Pediatric Research Institute, Beijing Children's Hospital, Capital Medical University, National Center for Children's Health; ${ }^{2}$ Beijing Key Laboratory of Pediatric Hematology Oncology; National Key Discipline of Pediatrics, Capital Medical University; Key Laboratory of Major Diseases in Children, Ministry of Education; Hematology Oncology Center, Beijing Children's Hospital, Capital Medical University, National Center for Children's Health and 'Beijing Advanced Innovation Center for Big Data-Based Precision Medicine, Beihang University and Capital Medical University, Beijing, China

Correspondence: RUI ZHANG - ruizh1973@126.com

TIAN-YOUWANG - wangtianyou@bch.com.cn

ZHI-GANGLI-ericlzg70@hotmail.com

doi:10.3324/haematol.2019.229187

Acknowledgments: the authors would like to thank the Special Fund of The Pediatric Medical Coordinated Development Center of Beijing
Hospitals Authority (No. XTZD20180201), the Pediatric Project of Ai You Foundation (No. AYEK201802), the National Science and

Technology Key Projects (No. 2017ZX09304029003), and Beihang

University \& Capital Medical University Advanced Innovation Center for Big Data-Based Precision Medicine Plan (BHME-201912).

\section{References}

1. Allen CE, Merad M, McClain KL. Langerhans-Cell Histiocytosis. N Engl J Med. 2018;379(9):856-868.

2. Badalian-Very G, Vergilio J-A, Degar BA, et al. Recurrent BRAF mutations in Langerhans cell histiocytosis. Blood. 2010;116(11):19191923.

3. Héritier S, Emile JF, Barkaoui MA, et al. BRAF mutation correlates with high-risk Langerhans cell histiocytosis and increased resistance to first-line therapy. J Clin Oncol. 2016;34(25):3023-3030.

4. Héritier S, Hélias-Rodzewicz Z, Lapillonne H, et al. Circulating cellfree BRAF V600E as a biomarker in children with Langerhans cell histiocytosis. Br J Haematol. 2017;178(3):457-467.

5. Hyman DM, Diamond EL, Vibat CR, et al. Prospective blinded study of BRAF V600E mutation detection in cell-free DNA of patients with systemic histiocytic disorders. Cancer Discov. 2015;5(1):64-71.

6. Haupt R, Minkov M, Astigarraga I, et al. Langerhans cell histiocytosis (LCH): guidelines for diagnosis, clinical work-up, and treatment for patients till the age of 18 years. Pediatr Blood Cancer. 2013;60(2):175184.

7. Allen CE, Ladisch S, McClain KL. How I treat Langerhans cell histiocytosis. Blood. 2015;126(1):26-35.

8. Gadner H, Minkov M, Grois N, et al. Therapy prolongation improves outcome in multisystem Langerhans cell histiocytosis. Blood. 2013;121(25):5006-5014

9. Corcoran RB, Chabner BA. Application of Cell-free DNA analysis to cancer treatment. N Engl J Med. 2018;379(18):1754-1765.

10. Egeler RM, Katewa S, Leenen PJ, et al. Langerhans cell histiocytosis is a neoplasm and consequently its recurrence is a relapse: In memory of Bob Arceci. Pediatr Blood Cancer. 2016;63(10):1704-1712.

11. Donadieu J, Larabi IA, Tardieu M, et al. Vemurafenib for refractory multisystem Langerhans cell histiocytosis in children: an International Observational Study. J Clin Oncol. 2019;37(31):28572865.

12. Schwentner R, Kolenová A, Jug G, et al. Longitudinal assessment of peripheral blood BRAF V600E levels in patients with Langerhans cell histiocytosis. Pediatr Res. 2019;85(6):856-864.

13. Kolenová A, Schwentner R, Jug G, et al. Targeted inhibition of the MAPK pathway: emerging salvage option for progressive life-threatening multisystem LCH. Blood Adv. 2017;1(6):352-356.

14. Berres ML, Lim KP, Peters T, et al. BRAF-V600E expression in precursor versus differentiated dendritic cells defines clinically distinct LCH risk groups. J Exp Med. 2014;211(4):669-683. 